\title{
Hybrid Gaussian Point-Process Model for Finer Control of Myoelectric Robotic Hands
}

\author{
Sohail Siadatnejad, Francesco Negro, and Luca Citi
}

\begin{abstract}
This paper presents a novel mathematical approach to decode information from neural spike trains recorded using high-density surface EMG for the control of a robotic hand. During precision grasps characterised by low firing rates, the algorithm uses a point-process approach to extract the information encoded in the spike times. When performing power grasps, the increased firing rate makes the point-process less reliable and the algorithm automatically and gradually switches to an approach based on the EMG power. Unlike current point-process decoding algorithm relying on a Gaussian approximations of the observation model, this model adopts a fully Gaussian state-space representation thanks to a parametrization of the rate of an inverse Gaussian distribution and by modelling the inter-spike interval directly.
\end{abstract}

\section{INTRODUCTION}

The functionality of conventional myoelectric control systems is constrained by the limited amount of neural information that can be extracted from a small number of surface electrodes. This paper proposes a novel algorithm for the combined use of high-density surface EMG (hd-sEMG) and statistical models based on point processes to decode neuro-muscular control signals.

Statistical models based on point processes have received significant attention in the neuroscience community because of their ability to significantly improve neural decoding tasks [1], [2]. A prerequisite for the application of these neural decoding algorithms is the possibility of detecting and isolating the spike times of individual neurons (spike sorting), a process that has traditionally required invasive neural recordings. The use of hd-sEMG opens up the possibility of accessing single motor unit action potentials (MUAPs) in a noninvasive way [3] and therefore allows for the exciting opportunity of applying temporal decoding to the myoelectric control of robotic hands. Unfortunately, in case of intense contraction, the increased firing rate is likely to cause higher background noise and spike overlap, thus making an approach based on point processes unreliable. The algorithm presented here is hybrid, i.e. it uses both hd-sEMG-extracted spikes as well as EMG power as observations for decoding. The decoder has

This research was funded by the UK Engineering and Physical Sciences Research Council (grant number EP/N031806/1) and by the EU's Horizon 2020 research and innovation programme under the Marie Skłodowska-Curie (grant agreement No 702491, NeuralCon).

S. Siadatnejad and L. Citi are with the School of Computer Science and Electronic Engineering, University of Essex, Colchester, UK (Corresponding author: 1citi@ieee.org).

F. Negro is with the Università degli Studi di Brescia, Brescia, Italy. an adaptive "attention" parameter to gradually switch between these two modes of operation depending on the characteristics of the recorded signal.

A number of methods for extracting information from spike trains based on state-space representations of point processes have been successfully applied to a wide range of neural decoding problems [1], [2], [4]. These algorithms generally implement approximate Bayesian filters based on Gaussian approximations of the observation model [5], which is typically an inhomogeneous Poisson process with a logarithmic parametrization of the conditional intensity function. As a result, the posterior state probability is only approximately Gaussian and this approximation can propagate to all future prior and posterior state distributions. Conversely, this work adopts a novel approach to the extraction of information from neural spike trains based on a fully Gaussian state-space representation. This is achieved through a parametrization of the rate of an inverse Gaussian distribution and by modelling the inter-spike interval directly, rather than through an inhomogenous Poisson process. The algorithm is effectively a Kalman filter that incorporates every new observed event through a conventional Kalman Gaussian update. When an event is not fully observed (censoring), a Gaussian approximation is performed. Since this censored update is only local to the current time step, the approximation does not propagate to future states.

\section{ALGORITHM}

We model the kinematics (or dynamics) of a set of degrees of freedom (DoFs) through the evolution of a hidden stochastic state vector $\mathbf{x}_{t}$, which depends on the value of the DoFs of interest at time $t$ and possibly on their recent past. We assume that this hidden state $\mathbf{x}_{t}$ evolves over time (sampled at, e.g., $1 \mathrm{~ms}$ ) as:

$$
\operatorname{Pr}\left(\mathbf{x}_{t} \mid \mathbf{x}_{t-1}\right)=\mathrm{N}\left(\mathbf{x}_{t} ; \mathbf{A}_{t} \mathbf{x}_{t-1}, \mathbf{Q}\right),
$$

and that we can indirectly observe it through measurements of the EMG power $\mathbf{v}_{t}$ and through MUAPs obtained applying ICA and spike sorting to the hdsEMG signal. The spike trains of MUAPs corresponding to unit $i$ are represented through the corresponding sample path $N_{t}^{i}$, i.e. the stochastic process counting the number of spikes occurred up to time $t$ included [1]. The EMG power and the spike trains are considered conditionally independent: $\mathbf{v}_{t} \Perp \Delta N_{t}^{1} \Perp \ldots \Perp \Delta N_{t}^{n_{\mathrm{S}}} \mid \mathbf{x}_{t}$. 
We assume that the distribution of the inter-spike intervals follows an inverse Gaussian distribution using the parametrization $\operatorname{IG}_{\delta}(\cdot ; \delta, \kappa)$ suggested by Whitmore [6], which is stated directly in terms of the drift $\delta \in \mathbb{R}$ :

$$
\operatorname{IG}_{\delta}(y ; \delta, \kappa)=\sqrt{\frac{\kappa}{2 \pi y^{3}}} \exp \left(-\frac{\kappa(y \delta-1)^{2}}{2 y}\right)
$$

In our case, we set:

$$
\operatorname{Pr}\left(\Delta N_{t}^{i}=1 \mid \mathbf{x}_{t}\right) \propto \operatorname{IG}_{\delta}\left(t-t_{*}^{i}(t-1) ; \mathbf{w}^{i} \mathbf{x}_{t}, \kappa\right)^{\alpha_{\mathrm{S}}(t)},
$$

where $\mathbf{w}^{i}$ is a row vector of regression coefficients, $\alpha_{\mathrm{S}}(t)$ is the "attention" parameter for the point-process observations and $t_{*}^{i}(t)$ is a function returning the time of the last event occurred before or at time $t$.

Finally, we model the power $\mathbf{v}_{t}$ of a set of EMG signals using a log-normal distribution:

$$
\operatorname{Pr}\left(\log \mathbf{v}_{t} \mid \mathbf{x}_{t}\right) \propto N\left(\log \mathbf{v}_{t} ; \mathbf{B} \mathbf{x}_{t}, \mathbf{S}\right)^{\alpha_{\mathrm{P}}(t)},
$$

where $\alpha_{\mathrm{P}}(t)$ is the "attention" parameter for the EMG power observations while $\mathbf{B}$ and $\mathbf{S}$ are the observation and covariance matrix of the power observations.

Importantly, both (3) and (4) represent quadratic loglikelihoods in $\mathbf{x}_{t}$, meaning that the assimilation of a fully observed spike interval or of a power measurement will preserve the Gaussianity of the state:

$$
\operatorname{Pr}\left(\mathbf{x}_{t} \mid \mathbf{v}_{1: t}, N_{1: t_{*}^{1}(t)}^{1}, \ldots, N_{1: t_{*}^{n}(t)}^{n_{\mathrm{S}}}\right)=\mathrm{N}\left(\mathbf{x}_{t} ; \hat{\mathbf{x}}_{t \mid t}^{*}, \mathbf{P}_{t \mid t}^{*}\right),
$$

where $\hat{\mathbf{x}}_{t \mid t}^{*}$ and $\mathbf{P}_{t \mid t}^{*}$ represent the posterior mode and covariance at time $t$ after accounting for all power measurements up to time $t$ and the sample paths of all spike trains up to their last observed spike. Finally, the full posterior probability of $\mathbf{x}_{t}$ can be written as:

$\operatorname{Pr}\left(\mathbf{x}_{t} \mid \mathbf{v}_{1: t}, N_{1: t}^{1: n_{\mathrm{S}}}\right) \propto \mathrm{N}\left(\mathbf{x}_{t} ; \hat{\mathbf{x}}_{t \mid t}^{*}, \mathbf{P}_{t \mid t}^{*}\right) \prod_{i=1}^{n_{\mathrm{S}}} \mathcal{R}\left(t-t_{*}^{i}(t) ; \mathbf{w}^{i} \mathbf{x}_{t}, \kappa\right)^{\alpha_{\mathrm{S}}(t)}$

where $\mathcal{R}(y ; \delta, \kappa)$ is the survival or reliability function of the $\mathrm{IG}_{\delta}$ distribution. If desired, a Laplace approximation $\mathrm{N}\left(\mathbf{x}_{t} ; \hat{\mathbf{x}}_{t \mid t}, \mathbf{P}_{t \mid t}\right)$ of this posterior can be obtained.

Effectively, our algorithm estimates the hidden state (and thus the desired DoFs) by iterating through the following steps: a) compute the one-step prediction $\hat{\mathbf{x}}_{t \mid t-1}^{*}$ from $\hat{\mathbf{x}}_{t-1 \mid t-1}^{*}$ using (1); b) combine the current a priori prediction $\hat{\mathbf{x}}_{t \mid t-1}^{*}$ with the current observation of the power measurements and any observed spikes to compute the a posteriori state estimate $\hat{\mathbf{x}}_{t \mid t}^{*}$ according to $(3)-(5)$; c) refine the a posteriori state estimate using the censored observations to obtain $\hat{\mathbf{x}}_{t \mid t}$ using (6).

\section{RESULTS}

We present preliminary tests of the algorithm on simulated data.
1) Generation of the simulated signals: Two motor neuron pools were simulated: flexor and extensor. Each pool included 150 motor neurons, each one simulated by a leaky integrate-and-fire model with discharge characteristics similar to [7]. The twitch forces of the motor units were selected with an exponential variation of the peak-to-peak amplitude and contraction times. Forces were normalized according to the maximal force (MVC) generated by the model, producing a number of simulated contractions in the range $0-10 \%$ MVC. A $17 \times 11$ matrix of hd-sEMG signals was simulated as convolution and summation of each motor neuron spike train with multichannel MUAPs derived by a cylindrical model [8]. White noise was added to each channel to simulate electrode and amplifier noise.

2) Decoding: Spike trains from two neurons (one per pool) were contaminated with Poisson-distributed spurious spikes and used for the decoding together with the power signals computed from two sources reconstructed by applying ICA to the simulated hdsEMG. These were used as observations $\left(N_{t}^{1}, N_{t}^{2}\right.$, and $\mathbf{v}_{t}$ ) by the model described in Section II to infer the hidden state $\mathbf{x}_{t}$ and ultimately the force produced. For simplicity $\alpha_{\mathrm{S}}(t)$ and $\alpha_{\mathrm{P}}(t)$ were kept constant in this preliminary analysis. The root mean squared error between the estimated and actual force was $1.87 \%$ MVC when only the two spikes trains were used, $1.67 \%$ MVC when only the power signals were used, and $1.46 \%$ MVC when combining spikes trains and power according to the model presented.

\section{CONCULSIONS}

The model combining spikes and power improves over the use of either one of the two types of signal. We speculate that the improvement will be even greater if the "attention" parameters are allowed to vary over time depending on the characteristics of the signals.

\section{REFERENCES}

[1] E. N. Brown, L. M. Frank et al., "A statistical paradigm for neural spike train decoding applied to position prediction from ensemble firing patterns of rat hippocampal place cells," $J$. Neurosci., vol. 18, no. 18, pp. 7411-7425, 1998.

[2] L. Paninski, Y. Ahmadian et al., "A new look at state-space models for neural data," J. Comput. Neurosci., vol. 29, no. 1-2, pp. 107126, 2010.

[3] D. Farina and F. Negro, "Accessing the neural drive to muscle and translation to neurorehabilitation technologies," IEEE Rev. Biomed. Eng., vol. 5, pp. 3-14, 2012.

[4] R. Barbieri, M. Wilson et al., "An analysis of hippocampal spatiotemporal representations using a Bayesian algorithm for neural spike train decoding," IEEE Trans. Neural Syst. Rehabil. Eng., vol. 13, no. 2, pp. 131-136, 2005.

[5] S. Koyama, U. T. Eden et al., "Bayesian decoding of neural spike trains," Ann. Inst. Stat. Math., vol. 62, no. 1, pp. 37-59, 2010.

[6] G. A. Whitmore, "A regression method for censored inverseGaussian data," Can. J. Stat., vol. 11, no. 4, pp. 305-315, 1983.

[7] F. Negro and C. Orizio, "Robust estimation of average twitch contraction forces of populations of motor units in humans," $J$. Electromyogr. Kinesiol., vol. 37, pp. 132 - 140, 2017.

[8] F. Negro, K. Keenan, and D. Farina, "Power spectrum of the rectified EMG: when and why is rectification beneficial for identifying neural connectivity?" J. Neural Eng., vol. 12, no. 3, p. $036008,2015$. 